\title{
Relative height error analysis of TanDEM-X elevation data
}

\author{
Paola Rizzoli*, Benjamin Bräutigam, Thomas Kraus, Michele Martone, Gerhard Krieger \\ Microwaves and Radar Institute, German Aerospace Center (DLR), Oberpfaffenhofen, Germany
}

\section{A R T I C L E I N F O}

Article history:

Available online 9 July 2012

\section{Keywords:}

SAR interferometry

Digital elevation model

Relative height error

TanDEM-X

\begin{abstract}
A B S T R A C T
The primary objective of the TanDEM-X mission is the generation of a global high resolution digital elevation model (DEM) with single-pass SAR interferometry. Within the mission, the Earth's land masses will be mapped at least twice to achieve relative vertical accuracies in the order of two meters. This paper presents an analysis of the mission performance in terms of the relative height error showing first results obtained from TanDEM-X interferometric data. For critical areas characterized by strong volume decorrelation phenomena or mountainous terrain, different approaches to improve the final height error are investigated as well.

(c) 2012 International Society for Photogrammetry and Remote Sensing, Inc. (ISPRS) Published by Elsevier
\end{abstract} B.V. All rights reserved.

\section{Introduction}

TerraSAR-X (launched in June 2007) and TanDEM-X (launched in June 2010) are two German SAR satellites, developed within a public/private partnership between the German Aerospace Center (DLR) and EADS Astrium. Both satellites serve for two different SAR missions: the TerraSAR-X mission (Werninghaus and Buckreuss, 2010), where both satellites provide high-quality SAR products for the science community and commercial purposes, and the TanDEM-X mission (Krieger et al., 2007) (started in June 2010) which has the primary goal of generating a global, high precision digital elevation model (DEM). Since October 2010, both satellites have been flying in close orbit configuration, enabling the acquisition of highly accurate cross- and along-track interferograms, which will be used as starting point for the generation of DEMs using single-pass SAR interferometry techniques (Krieger et al., 2007; Fritz et al., 2011). Digital elevation models are required for many purposes, such as navigation, cartography and the orthorectification of other remote sensing image products. Up to now, DEMs have been derived using several SAR sensors, both airborne and spaceborne. Available global DEMs are the GTOPO30 (USGS GTOPO, 2001), provided by the United States Geological Survey (USGS) and characterized by a horizontal grid spacing of $30^{\prime \prime}$ (approximately $1 \mathrm{~km}$ ), and the one provided by the Shuttle Radar Topography Mission (SRTM) (Werner, 2001), whose coverage is limited to a latitude range between $56^{\circ} \mathrm{S}$ and $60^{\circ} \mathrm{N}$ and which is delivered with a grid spacing of $1^{\prime \prime}$ for regions within the US territory and less than $3^{\prime \prime}$ for the rest of the mapped areas. An overview of the SRTM mission performance can be found in (Rodriguez et al., 2006), where

\footnotetext{
* Corresponding author.

E-mail address: Paola.Rizzoli@dlr.de (P. Rizzoli).
}

different sources of errors, such as baseline determination, thermal noise, timing and position errors and beam overlapping mismatches are taken into account. Three different types of errors characterize the final DEM: an absolute height error, which can be properly calibrated using ground reference targets, a random error, which is caused by thermal noise and residual geometric decorrelation effects, and geolocation errors due to instrument time and phase offsets, which can be properly calibrated by using ground control points like corner reflectors. These three error sources are taken into account to define the TanDEM-X DEM product specification, as presented in (Wessel, 2011), leading to the definition of the following three quantities: absolute vertical accuracy, relative vertical accuracy and absolute horizontal accuracy. The specified values are presented in Table 1 . In this paper, only the relative vertical accuracy will be addressed.

The TanDEM-X system was commissioned during a period of about 5 months after the launch (Hueso Gonzalez et al., 2010). The single TanDEM-X satellite was first calibrated to fulfill the TerraSAR-X mission requirements, acquiring data in monostatic configuration (Kraus et al., 2011; Schwerdt et al., 2011). The bistatic performance was then analyzed in detail, taking into account different acquisition geometries, modes and radar parameters (Martone et al., 2011). The mission operational phase started in December 2010, with the aim of providing a complete high-resolution DEM of the Earth land masses in 2014. The nominal operation mode for the systematic data acquisition is the bistatic Stripmap mode, where both satellites offer the flexibility to work as the master transmitting sensor in order to equally share the power resources. To provide a good interferometric basis for multi-baseline phase unwrapping, a first complete coverage of the land masses has been achieved after the first year of operation, acquiring interferograms with a target height of ambiguity ( $\mathrm{HoA})$ higher than 
Table 1

TanDEM-X DEM specifications.

\begin{tabular}{lll}
\hline Requirement & Specification & Accuracy \\
\hline Relative vertical accuracy & $\begin{array}{l}90 \% \text { linear point-to point } \\
\text { error over a } 1^{\circ} \times 1^{\circ} \text { cell }\end{array}$ & $2 \mathrm{~m}($ slope $<20 \%)$ \\
Absolute vertical accuracy & $90 \%$ linear error & $4 \mathrm{~m}($ slope $>20 \%)$ \\
Absolute horizontal accuracy & $90 \%$ circular error & $10 \mathrm{~m}$ \\
Spatial resolution & independent pixels & $12 \mathrm{~m}$ \\
& & $\left(0.4^{\prime \prime}\right.$ at equator $)$ \\
\hline
\end{tabular}

$45 \mathrm{~m}$. The $H o A$ is defined as the height difference equivalent to a $2 \pi$ phase cycle inside an interferogram and can be expressed, for the bistatic case, as:

$H o A=\frac{\lambda r \sin \theta_{i}}{B_{\perp}}$

where $\lambda$ is the radar wavelength, $r$ the slant range, $\theta_{i}$ the incidence angle and $B_{\perp}$ the baseline perpendicular to the line of sight.

To meet the specifications on the final vertical accuracy in challenging areas, such as mountains and tall forests, additional data have to be acquired as needed. The final DEM is then generated by mosaicking all data acquisitions (Hueso Gonzalez et al., 2010).

This paper focuses on the analysis of the system performance in terms of the relative height error, showing first results derived from the acquired TanDEM-X elevation data. It is structured as follows: Section 2 presents the theoretical approach used for the estimation of the point-to-point relative height error. Section 3 shows several examples of the actual performance obtained over test sites characterized by different HoAs and land cover types and presents a comparison with the theoretical performance. Finally, Section 4 describes different approaches for monitoring the mission performance and improving the relative height error over critical areas.

\section{Relative height error estimation approach}

Given two SAR images acquired from displaced locations, it is possible to reconstruct the DEM of the ground starting from the phase interferogram between the two. Here, the relative height error is intended to describe white noise-like contributions inside the DEM, which cannot be removed by the final calibration and mosaiking process. The estimation of the relative height error is based on the quantification of the interferometric phase errors. The phase difference $\varphi$ between two interferometric SAR channels can be described as a random variable, characterized by its probability density function (pdf) $p_{\varphi}(\varphi)$ (Lee et al., 1994). It is related to the total interferometric coherence $\gamma_{t o t}$, which quantifies the error contributions coming from different sources, such as limited signal-tonoise ratio (SNR), volume and temporal decorrelation effects as well as range and azimuth ambiguities, as described in (Krieger et al., 2007). The estimation of the $90 \%$ point-to-point phase error $\Delta \varphi_{90 \%}$ requires the computation of the $90 \%$ percentile from the pdf obtained by evaluating the difference between two random variables, each of them describing the fluctuation of the interferometric phase within one interferogram (Krieger et al., 2007). For a considered $\mathrm{HoA}$, the point-to-point $90 \%$ relative height error is then derived from the interferometric phase error as:

$\Delta h_{90 \%}=H o A \cdot \frac{\Delta \varphi_{90 \%}}{2 \pi}$.

In the following, we describe an approach that has been implemented to directly estimate the point-to-point relative height error from real SAR data, taking into account two successively acquired DEMs $D_{1}\left(\mathbf{r}_{1}\right)$ and $D_{2}\left(\mathbf{r}_{2}\right)$. The two DEMs are acquired with the same acquisition parameters and over mutually overlapping regions of interest $\mathbf{r}_{1}$ and $\mathbf{r}_{2}$ in [lat/lon] coordinates. Moreover, both DEMs are supposed to be affected by independent noise components. A common region of interest $\mathbf{r}$ has to be defined as $\mathbf{r}=\mathbf{r}_{1} \cap \mathbf{r}_{2}$, so that both DEMs can be referred to the same ground coordinates, leading to $D_{1}(\mathbf{r})$ and $D_{2}(\mathbf{r})$. The reference frame can now be transformed from [lat/lon] coordinates to horizontal and vertical distances by evaluating the single coordinate distances on the WGS84 ellipsoid. A further interpolation is required in order to obtain a uniform grid of space coordinates $[x, y]$, whose orthogonal spacings are defined as $\Delta x$ and $\Delta y$.

Starting from $D_{1}(x, y)$ and $D_{2}(x, y)$, the DEM difference can now be evaluated:

$\Delta D(x, y)=D_{1}(x, y)-D_{2}(x, y)$.

Systematic contributions due to an unprecise estimation of acquisition parameters, such as sensor orbit position and baseline, produce slowly varying errors within a SAR DEM and will be compensated during the final DEM calibration process. Heance, a high-pass filtering is required to remove such slowly varying components and quantify the relative height error from the DEM difference. The filtering process can be performed in the frequency domain as explained in the following. The two-dimensional Fourier transform of the DEM difference $\Delta D(x, y)$ is evaluated as:

$S\left(k_{x}, k_{y}\right)=\int_{0}^{y_{0}} \int_{0}^{x_{0}} \Delta D(x, y) e^{-j k_{x} x} e^{-j k_{y} y} d x d y$,

where $k_{x}$ and $k_{y}$ are the spatial frequency domain variables. The maximum spatial coordinates $x_{0}$ and $y_{0}$ are defined as $x_{0}=N_{x} \Delta x$, $y_{0}=N_{y} \Delta y, N_{x}$ and $N_{y}$ being the number of samples in the horizontal and vertical dimensions. A two-dimensional circular high-pass filter $F^{\text {high }}\left(k_{x}, k_{y}\right)$ is designed in the frequency domain as:

$F^{\text {high }}\left(k_{x}, k_{y}\right)=1-F^{\text {low }}\left(k_{x}, k_{y}\right)$,

$F^{\text {low }}\left(k_{x}, k_{y}\right)$ being a two-dimensional low-pass gaussian filter, defined as:

$F^{l o w}\left(k_{x}, k_{y}\right)=e^{\frac{-\left(k_{x}^{2}+k_{y}^{2}\right)}{2\left(\sigma_{x}^{2}+\sigma_{y}^{2}\right)}}$

where $\sigma_{x}^{2}, \sigma_{y}^{2}$ describe the bandwidth in the horizontal and vertical dimensions. The filter bandwidth has been experimentally chosen by finding a compromise between an eventual error underestimation and the undesired permanency of low-frequency components in the filtered data, leading to a cut-off frequency of about $1 / 10$ of the whole bandwidth. The high-pass filtering operation is then performed by

$S^{h i g h}\left(k_{x}, k_{y}\right)=S\left(k_{x}, k_{y}\right) F^{h i g h}\left(k_{x}, k_{y}\right)$

and the estimated relative height error is retrieved as:

$\Delta h(x, y)=\frac{1}{4 \pi^{2}} \int_{-\frac{k_{0}}{2}}^{\frac{k_{x_{0}}}{2}} \int_{-\frac{k_{0}}{2}}^{\frac{k_{y_{0}}}{2}} S^{h i g h}\left(k_{x}, k_{y}\right) e^{j x k_{x}} e^{j y k_{y}} d k_{x} d k_{y}$,

where $k_{x_{0}}$ and $k_{y_{0}}$ are defined as:

$k_{x_{0}}=\frac{2 \pi}{\Delta x}, \quad k_{y_{0}}=\frac{2 \pi}{\Delta y}$

The DEM specifications have to be verified for flat and mountainous terrain separately, as presented in Table 1 . In order to discriminate flat from mountainous areas, a slope mask can be generated by evaluating the local DEM gradient: starting from a smoothed version of one of the two input DEMs $D_{1}(x, y)$ or $D_{2}(x, y)$, the horizontal and vertical DEM gradient components are evaluated as:

$\nabla D_{1}(x, y)=\left(\frac{\partial D_{1}(x, y)}{\partial x}, \frac{\partial D_{1}(x, y)}{\partial y}\right)$ 


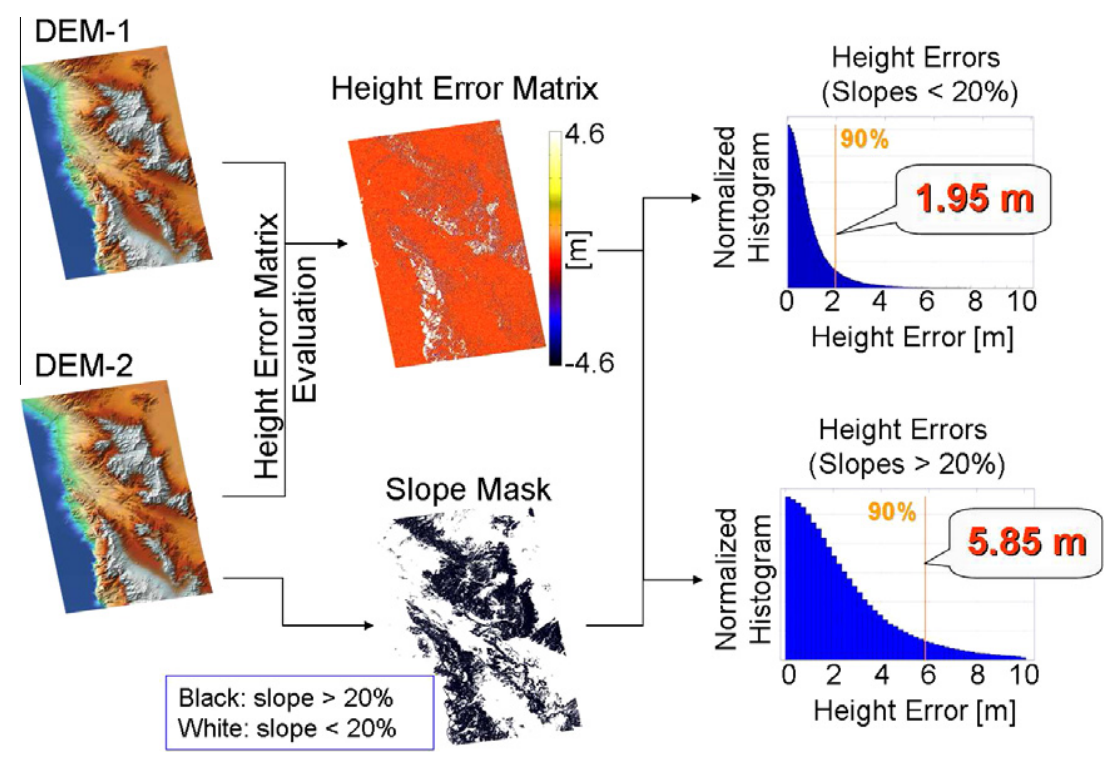

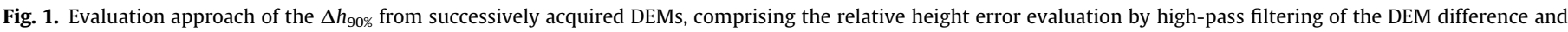

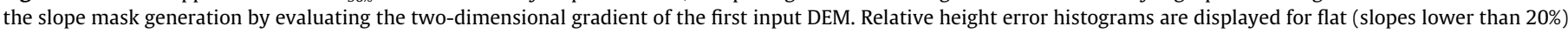
and mountainous terrain (slopes higher than 20\%). Here, soil and rocks acquisitions over Death Valley (USA) are displayed.

Then, the predominant local slope is retrieved by computing the Euclidean norm of the gradient vector:

$G(x, y)=\sqrt{\left(\frac{\partial D_{1}(x, y)}{\partial x}\right)^{2}+\left(\frac{\partial D_{1}(x, y)}{\partial y}\right)^{2}}$.

Height errors over flat and mountainous areas are discriminated by setting a threshold $T_{\text {slope }}$ on the local slope. The $\left[x_{f}, y_{f}\right]$ and $\left[x_{m}, y_{m}\right]$ coordinates over flat (index $f$ ) and mountainous (index $m$ ) areas are separately evaluated by imposing a threshold $T_{\text {slope }}$ on $\left|G\left(x_{f}, y_{f}\right)\right|$.

Since both positive and negative contributions have to be taken into account, the $90 \%$ height error $\Delta h_{90 \%}$ can be finally estimated by evaluating the $90 \%$ percentile from the histograms of $\left|\Delta h\left(x_{f}, y_{f}\right)\right|$ and $\left|\Delta h\left(x_{m}, y_{m}\right)\right|$. Fig. 1 shows the complete height error estimation chain, starting from two successively acquired DEMs.

\section{Point-to-point relative height error verification}

3.1. Analysis of the incidence angle dependence of the relative height error

For any two successively acquired DEMs, the height error behavior along the range dimension can be analyzed by dividing the relative height error matrix into blocks of about $600 \mathrm{~m}$ in the range dimension and averaging over the whole azimuth scene length. For each of them the $\Delta h_{90 \%}$ is evaluated. Fig. 2 shows on the left-hand side the $\Delta h_{90 \%}$ range profiles as a function of the incidence angles for six different acquisitions over soil and rocks terrain, according to the GLOBCOVER classification map (GLOBCOVER, 2009). Only flat terrain has been taken into account (the corresponding $H o A$ is displayed as well). Due to the lower antenna gain, $\Delta h_{90 \%}$ increases at the swath edges, while the best performance is achieved at mid range, where a higher antenna pattern
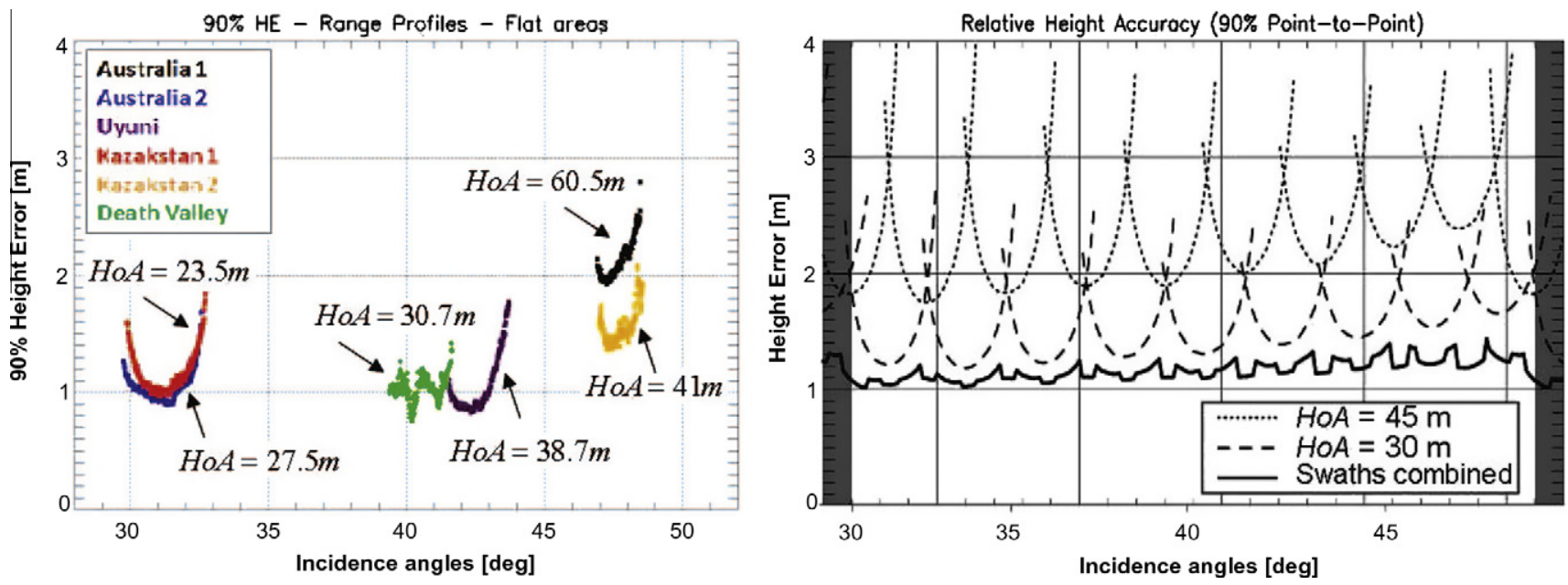

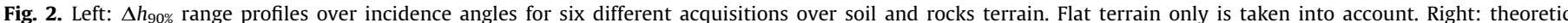

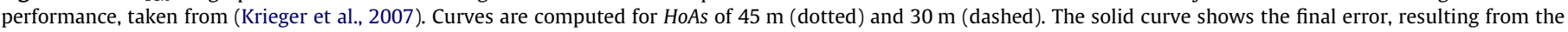
combination of multiple swaths, as explained in Section 4.2. 


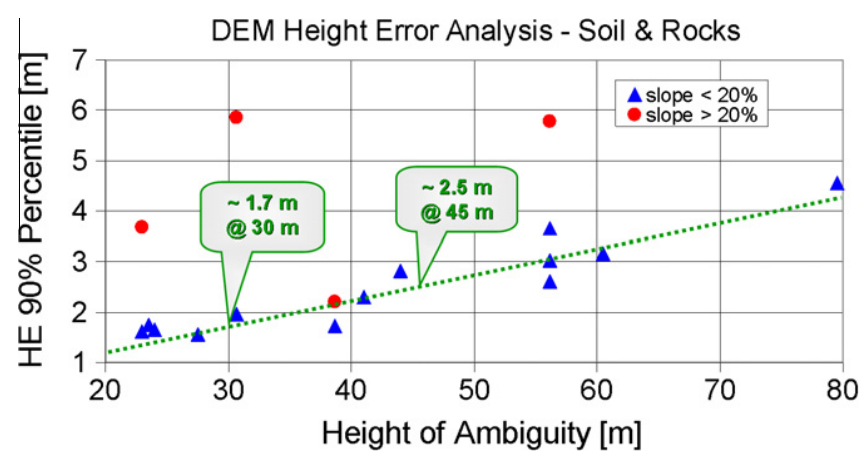

Fig. 3. Summary of the estimated $\Delta h_{90 \%}$ from TanDEM-X repeated acquisitions over soil and rocks terrain for different HoAs. A linear trend is fitted over $\Delta h_{90 \%}$ measurements for flat areas. Green boxes highlight the predicted performance from (Krieger et al., 2007) according to Fig. 2.

gain allows for better SNR. Note that, in the case of the Death Valley test site (green), characterized by a $H o A$ of $30.7 \mathrm{~m}$, the $\Delta h_{90 \%}$ range profile is noisier with respect to the other ones because of the limited number of available samples over flat areas. This site is mostly mountainous, especially at mid range, while all the other test sites are almost completely flat. On the right-hand side of Fig. 2 the theoretically predicted performance, taken from (Krieger et al., 2007), is displayed. The comparison between the two graphs shows that, for ground areas characterized by stable backscatter, the elevation data measurements are better than the predicted performance.
This result is mainly due to the very stable conditions of the chosen test sites and to the conservative assumption about the backscattering levels which has been taken into account in the predicted performance.

\subsection{Relative height error for different HoAs}

The theoretical dependency of the relative height error on the $H o A$ has been presented in Eq. (2). Fig. 3 shows a summary of the estimated $\Delta h_{90 \%}$ from TanDEM-X repeated acquisitions over soil and rocks terrain for different HoAs. Since many of the considered acquisitions are characterized by almost completely flat terrain, the $\Delta h_{90 \%}$ over mountainous areas is available for a few test sites only. A linear trend is fitted over the $\Delta h_{90 \%}$ measurements for flat areas, leading to an estimate of $\Delta h_{90 \%}$ equal to $1.7 \mathrm{~m}$ for an $H o A$ of $30 \mathrm{~m}$ and $\Delta h_{90 \%}$ equal to $2.5 \mathrm{~m}$ for an $\mathrm{HoA}$ of $45 \mathrm{~m}$. The two HoAs taken into account are the ones for which the theoretical point-topoint $\Delta h_{90 \%}$ profiles in Fig. 2 (right) are evaluated. These results match very well with the theoretical performance where, if swath overlapping areas are not taken into account, the $\Delta h_{90 \%}$ resides within $1.2 \mathrm{~m}$ and $2 \mathrm{~m}$ for an $\mathrm{HoA}$ of $30 \mathrm{~m}$, and within $1.8 \mathrm{~m}$ and $3 \mathrm{~m}$ for an $\mathrm{HoA}$ of $45 \mathrm{~m}$.

\subsection{Relative height error for different land cover classes}

The estimated relative height error depends not only on the system acquisition parameters, but also on the ground target characteristics. Illuminated areas can be grouped into different land cover
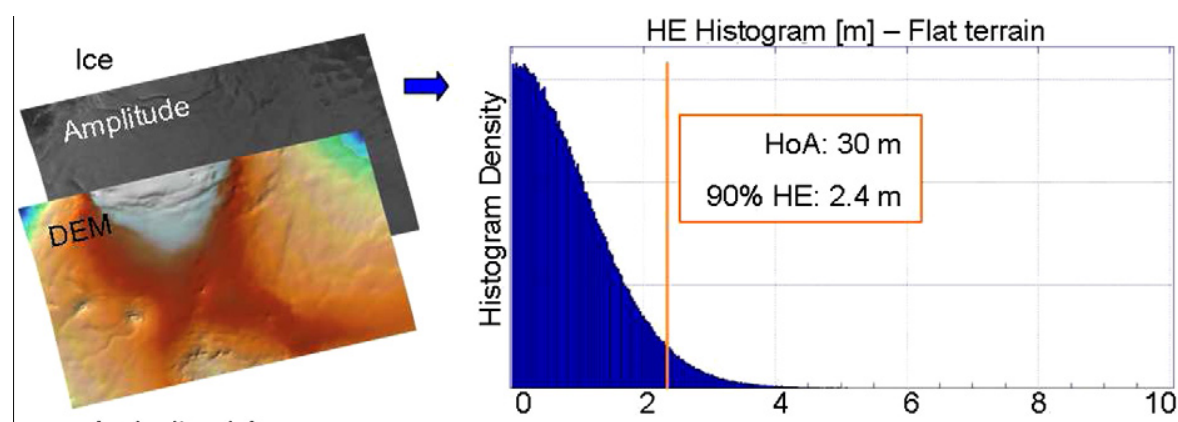

(a)
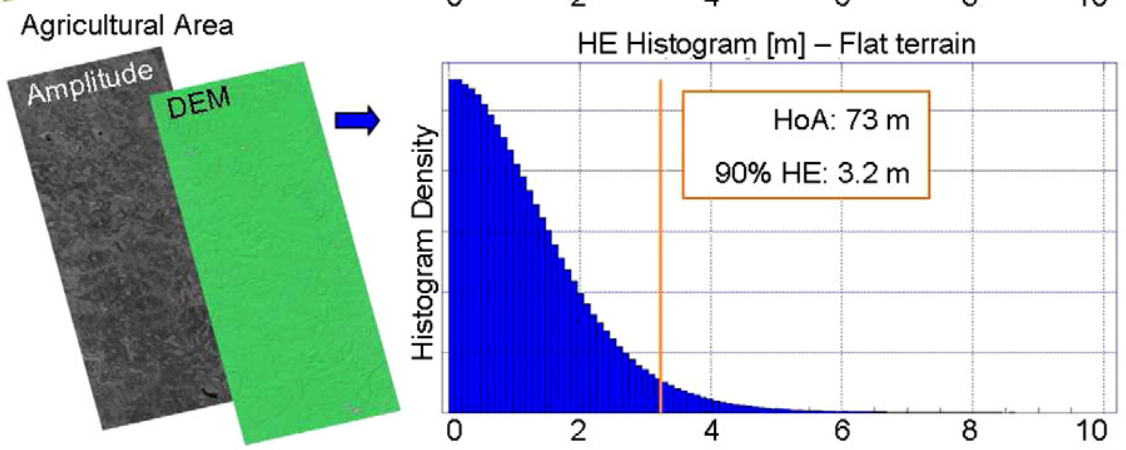

(b)
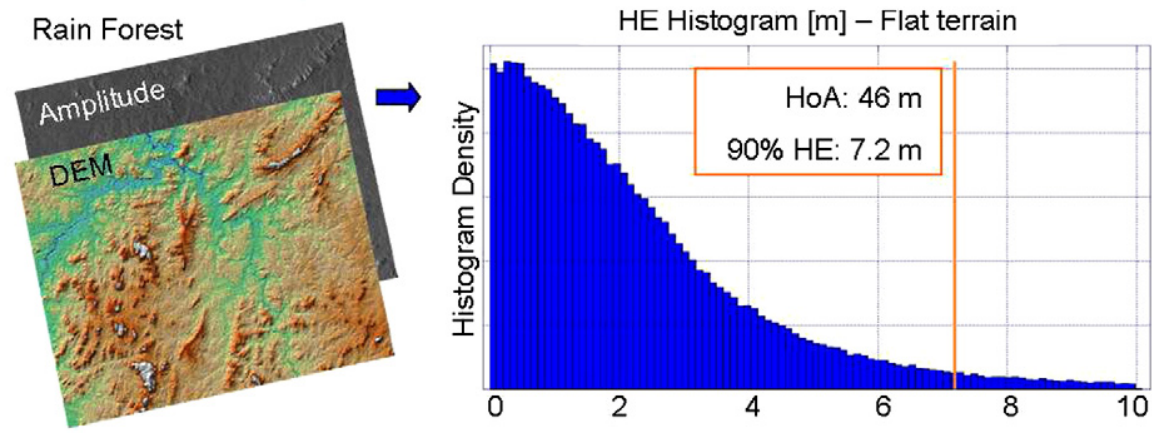

(c)

Fig. 4. $\Delta h_{90 \%}$ for different land cover classes: ice, agricultural areas and rain forest. 


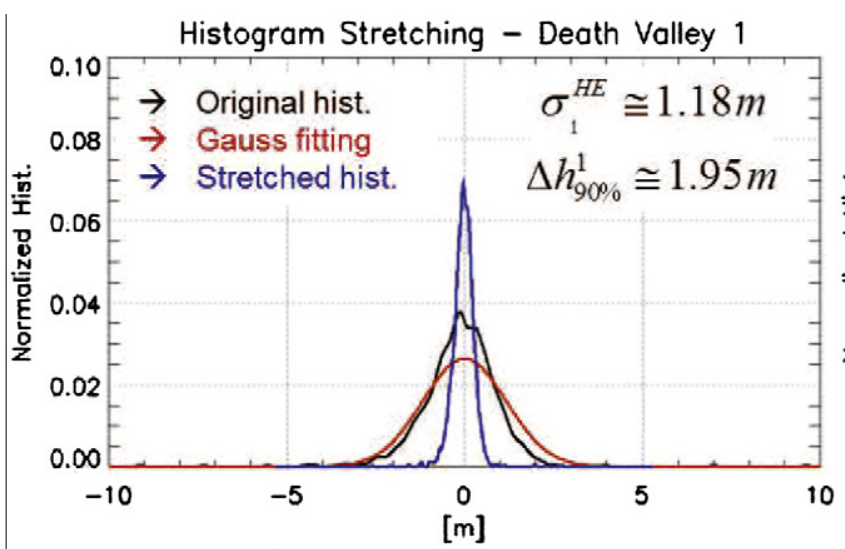

(a)

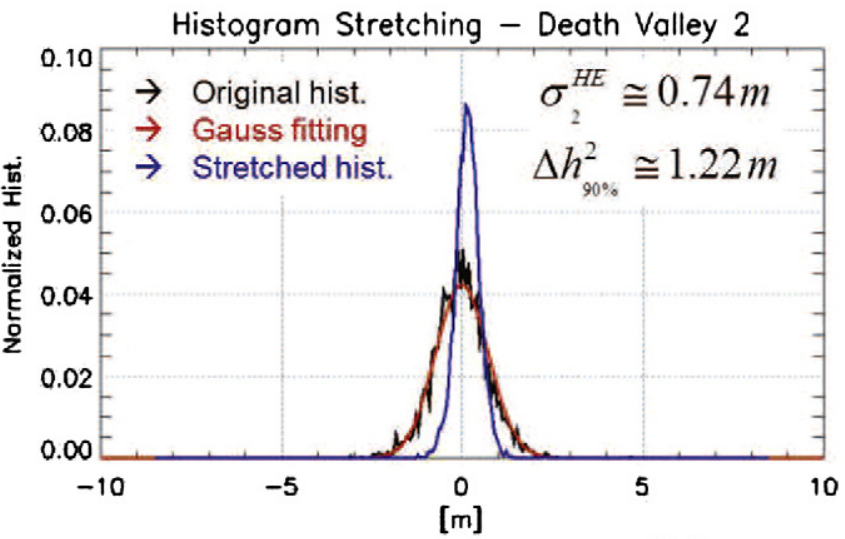

(b)

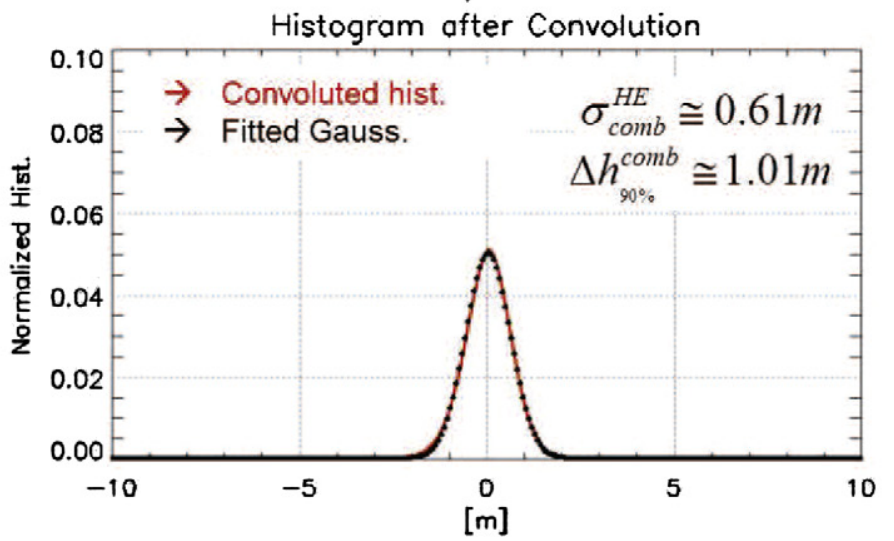

(c)

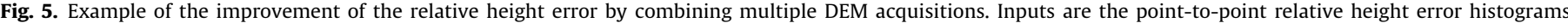

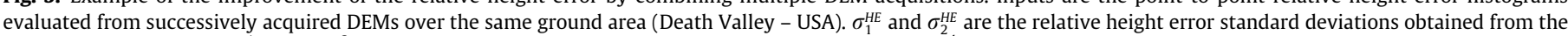

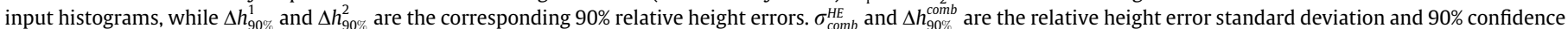
level after the convolution.

classes and separately analyzed, allowing for the development of different acquisition strategies for each of them, e.g. by setting different minimum HoAs as presented in Section 4.1. Fig. 4 shows an example of some of the considered test sites, characterized by the following land cover classes: ice (a), agricultural areas (b) and rain forest (c). Note that the same system parameters (i.e. baseline and $H o A$ ) are not available over different areas. For this reason, data acquired with different HoAs are used for performing the relative height error analysis for different land cover classes. It becomes clear that, for all cases, further acquisitions will be required in order to meet the final DEM specification. This will be presented in Section 4.2. Different aspects, such as volume and temporal decorrelation, have an additional impact on the estimation technique, so that results may not match with the theoretical performance. A particularly significant example is given by the estimated $\Delta h_{90 \%}$ over the rain forest (Fig. 4c), where volume decorrelation strongly affects the final performance.

\section{Height error improvement approaches}

The final TanDEM-X mission performance in terms of the relative height error will have to satisfy the specifications described in Table 1. For critical areas, characterized by strong volume decorrelation or mountainous terrain, the acquisition strategy has to be specially optimized. For this reason, different approaches to improve the final height error performance have been investigated: depending on the acquisition parameters and on the target characteristics of the illuminated ground area, the achieved performance can be improved by changing the acquisition geometry or by increasing the number of acquisitions, which are used to generate the final DEM (multi-baseline multi-image interferometry). In the following sections, different solutions are proposed.

\subsection{HoA impact on height error over highly vegetated areas}

Highly vegetated areas, such as rain forest, are affected by strong volume decorrelation phenomena. In such cases, an increase of the HoA allows for a reduction of the volume decorrelation impact on the interferometric coherence, leading to a reduction of the relative phase error. For this reason, a minimum required $H o A$ of about $45 \mathrm{~m}$ has been established for acquisitions over vegetated areas, in order to assure a sufficient quality for the phase unwrapping (Martone et al., this issue). From Eq. (2), it can be noticed that the HoA cannot be arbitrarily incremented, because of the mission specifications on the final vertical DEM accuracy. For this reason, the approach explained in Section 4.2 will be taken into account as well.

\subsection{Height error improvement with multiple acquisitions}

A further improvement of the relative height error accuracy can be achieved by combining multiple interferometric acquisitions 


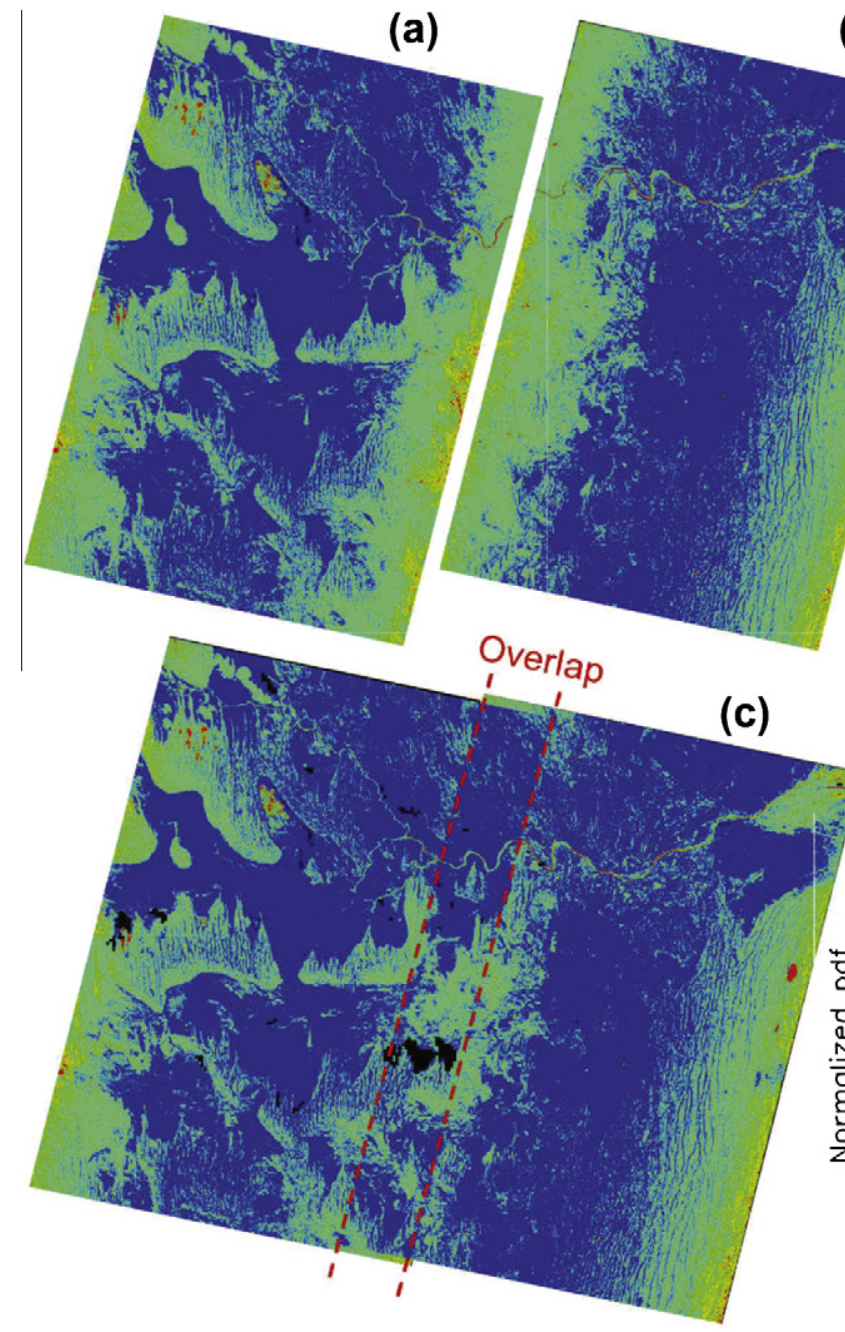

(b)

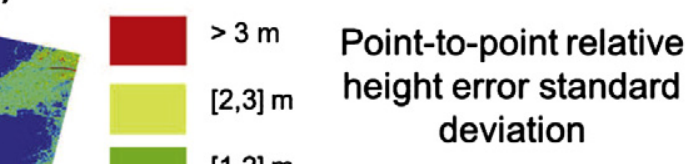

$[1,2] \mathrm{m}$

$[0,1] m$
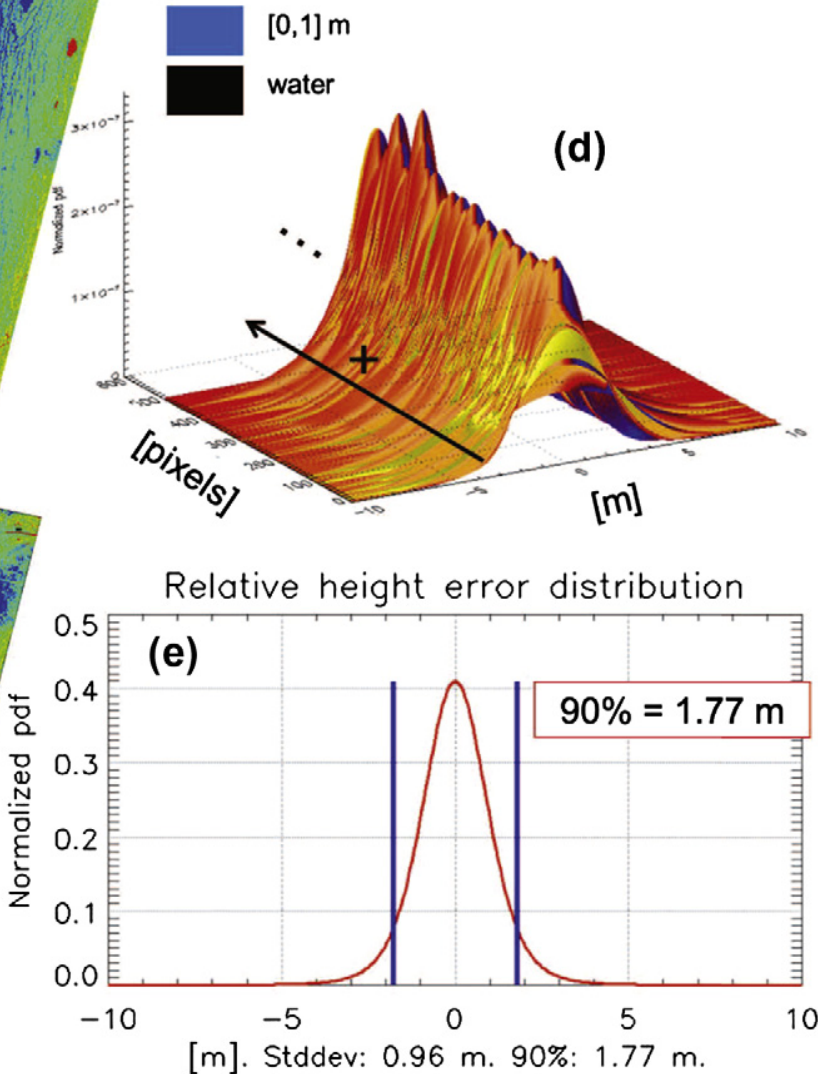

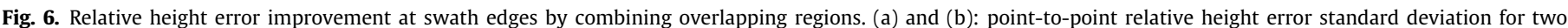

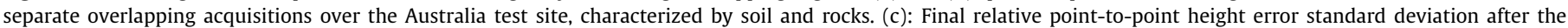

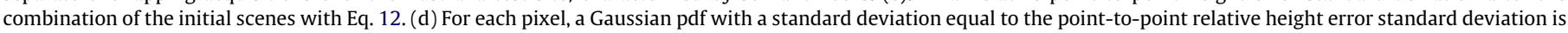
generated. (e) Pdf obtained from the sum of pdfs in (d) and estimation of the $\Delta h_{90 \%}$.

over the same ground area, recurring to multi-baseline phase unwrapping techniques, as described in (Lachaise et al., 2007). From Fig. 5 it can be inferred that the relative height error is reasonably well characterized by a Gaussian distribution (the Gaussian pdf, evaluated using the mean value and standard deviation retrieved from the histogram itself, is plotted as well). Knowing that the linear combination of two random variables with independent Gaussian distributions is still a random variable with Gaussian distribution, the final relative height error standard deviation $\Delta h_{\text {tot }}$, obtained by the optimum combination of multiple acquisitions, can be evaluated as (Krieger et al., 2007):

$\Delta h_{\text {tot }}=\sqrt{\frac{1}{\sum_{i=1}^{N} \frac{1}{\Delta h_{i}^{2}}}}$

where $N$ represents the total number of available acquisitions. Given the properties of a Gaussian distribution with zero mean, where the standard deviation and the $90 \%$ confidence level are related to each other by a fixed ratio, Eq. (12) can be applied to the $90 \%$ relative height error as well. This approach has been validated by comparing the obtained results with the ones generated by convolving a stretched version of the input histograms and evaluating the $\Delta h_{90 \%}$ of the obtained histogram. A stretching factor $\alpha_{i}$, evaluated as in (Krieger et al., 2007), has been applied to the ith input histogram. The example proposed in Fig. 5 presents the results obtained from such a test, taking into account two subsequent repeat-pass acquisitions over the same region of interest, situated over the Death Valley (USA), and characterized respectively by a $\mathrm{HoA}$ of about $39 \mathrm{~m}$ and $42 \mathrm{~m}$. Both acquisitions are characterized by Soil and Rocks ground classification and present a mean interferometric coherence higher than 0.8. Using the estimation approach presented in section 2 , the $\Delta h_{90 \%}$ over flat areas can be retrieved for each acquisition separately: $\Delta h_{90 \%}^{1}$ (sub-figure a) refers to the first acquisition, while $\Delta h_{90 \%}^{2}$ (sub-figure b) to the second one. A combined $90 \%$ height error $\Delta h_{90 \%}^{\text {comb }}$ of about $1.01 \mathrm{~m}$ is obtained after the convolution of the stretched histograms (sub-figure (c)), while the combination of the estimated $\Delta h_{90 \%}^{1}$ and $\Delta h_{90 \%}^{2}$ using Eq. (12) leads to a final $90 \%$ height error $\Delta h_{\text {tot }}$ of $1.02 \mathrm{~m}$. The obtained results are in good agreement with each other.

As can be inferred from Fig. 2, the worst performance is achieved at the swath edges. In order to assure the complete coverage of land masses, adjacent acquisitions are always characterized by an overlapped region, both at near and far range. In this specific area, two or more overlapping swaths are already available within the first global coverage, allowing for the improvement of 

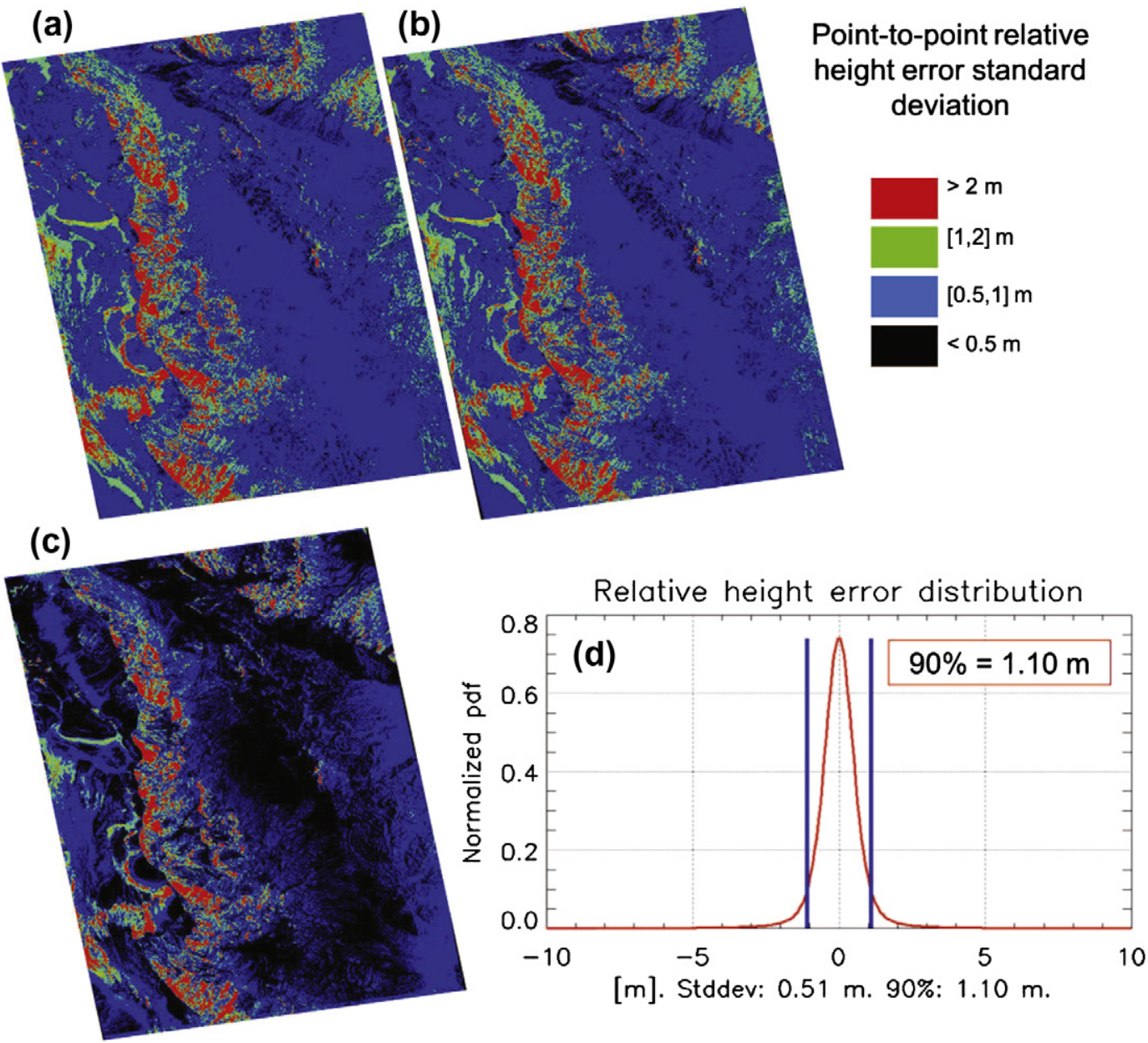

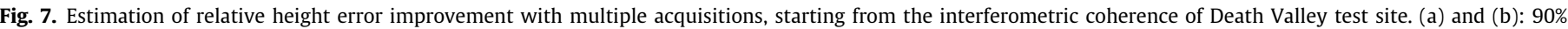

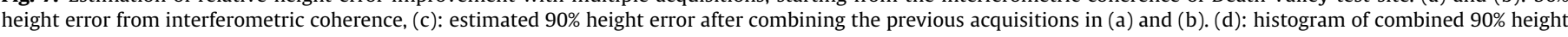
error per pixel.

the relative height error as explained above. In this way, in the case of highly coherent and stable areas (such as flat areas characterized by soil and rocks terrain) the combination of acquisitions from the first year of the TanDEM-X mission can be sufficient to achieve the required mission specification. An example is shown in Fig. 6. Two overlapping DEMs over Australia are taken into account: the single point relative height error standard deviation is firstly evaluated from the interferometric coherence, as presented in (Krieger et al., 2007). Assuming a zero mean normal distribution, as justified from the example in Fig. 5, the point-to-point relative height error standard deviation is then derived by multiplication with the square root of two. Fig. 6a and b present, for each input acquisition, the point-to-point relative height error standard deviation $\sigma^{H E}$ estimated from the interferometric coherence. Fig. 6c shows the final point-to-point relative height error standard deviation $\sigma_{\text {comb }}^{H E}$ obtained from the combination of the two initial scenes using Eq. (12). Images are color coded using different levels, each of them characterizing relative height errors comprised into definite ranges, as described by the colorbar. A consistent improvement of the performance is visible over the overlapped region, which involves the swath edges of both single acquisitions. The final pointto-point $\Delta h_{90 \%}$ is then directly estimated from $\sigma_{\text {comb }}^{\mathrm{HE}}$ in the following way: each pixel is supposed to be normally distributed with standard deviation $\sigma_{i}^{H E}$ and zero mean. The corresponding Gaussian pdf is evaluated for each available pixel (Fig. 6d) and the normalized sum of all the pdfs is evaluated. The $\Delta h_{90 \%}$ is finally retrieved from the resulting pdf, as presented in Fig. 6 e.

\subsection{Multiple acquisition global prediction approach}

The approach proposed in Section 4.2 shows a significant improvement of the relative height error performance using multiple acquisitions. In order to support the acquisition planning strategy for additional acquisitions, a global scale approach is introduced. It consists in combining multiple acquisitions using Eq. (12) and then deriving the 90\% point-to-point relative height error as presented in Section 4.2. This approach relies on available single-pass bistatic acquisitions only, instead of evaluating two or more DEM acquisitions which are available for dedicated test sites only. Fig. 7 presents in (a) and (b) the point-to-point relative height error standard deviation images, evaluated from each coherence matrix associated with two acquisitions over the Death Valley (USA), while Fig. 7c shows the combined point-to-point relative height error standard deviation. Sub-figure (d) on the right hand side shows the histogram of the combined height error standard deviation, evaluated as presented in section 4.2 and characterized by a $\Delta h_{90 \%}$ of $1.10 \mathrm{~m}$.

Note that areas characterized by a point-to-point relative height error higher than $2 \mathrm{~m}$ still remain. Such areas are mainly characterized by ground slopes higher than $20 \%$. The interferometric TanDEM-X performance parameters acquired up to now provide a solid basis for estimating the impact and necessity of multiple acquisitions on a global scale, depending on the on-ground topography, land coverage classification and acquisition parameters. An example is presented in Fig. 8: sub-figures (a) and (b) show respec- 

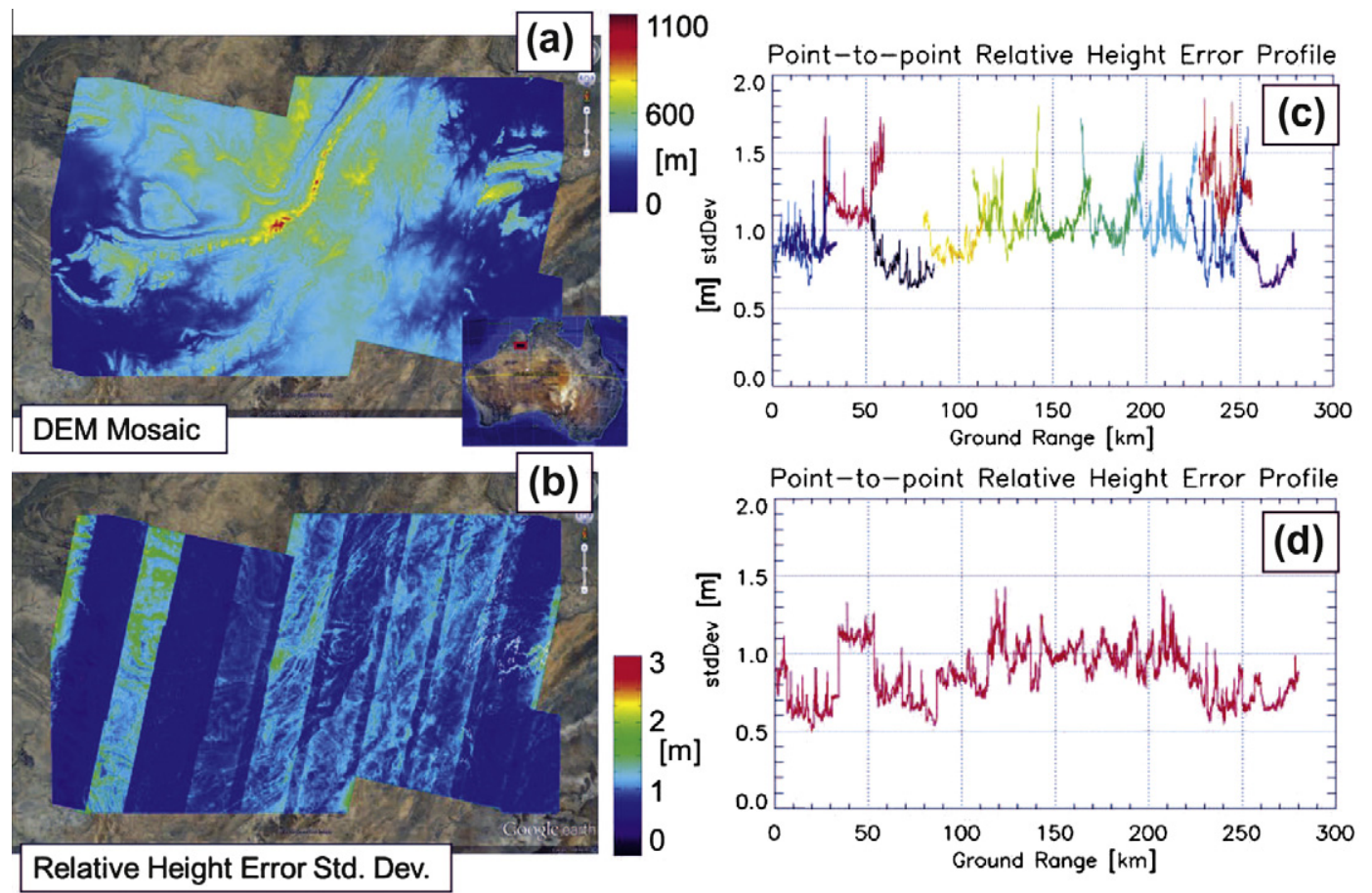

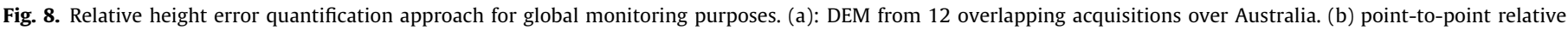

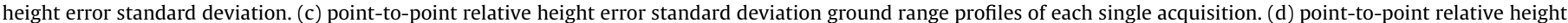
error standard deviation ground range profile of the composed acquisitions using Eq. (12).

tively the DEM and the point-to-point relative height error standard deviation $\sigma^{H E}$ obtained by mosaicking 12 different TanDEM$\mathrm{X}$ acquisitions over Australia, covering an area of about $250 \times 100 \mathrm{~km}^{2}$.

$\sigma^{H E}$ is obtained from the interferometric coherence, and Eq. (12) is applied in order to properly compose overlapping regions. Subfigure (c) presents, for each acquisition separately, the point-topoint relative height error standard deviation ground range profiles, evaluated by averaging the $\sigma^{H E}$ along the azimuth dimension. Note that the acquisition centered around a ground range of $50 \mathrm{~km}$ is characterized by higher height error, due to an higher $H o A$. Finally, sub-figure (d) shows the ground range $\sigma^{H E}$ profile after the combination of all the available acquisitions. It can be noticed that the $\sigma^{H E}$ is improved both at swath edges and where two acquisitions are available over the same ground area. The same approach can be repeated for different test sites, leading to a global overview of the system performance.

\section{Conclusions}

In this paper, the TanDEM-X system performance has been analyzed in terms of the relative height error. The estimation approach and the first results obtained from TanDEM-X successively acquired DEMs are presented. The relative height error depends on several factors, such as the ground characteristics and the acquisition geometry. Different tests have been analyzed, as presented in Sections 3.1, 3.2 and 3.3, showing the overall good performance of the TanDEM-X elevation data with a single acquisition already. Different approaches for improving the final DEM performance have been analyzed in Section 4, taking into account the consequences of changing the acquisition geometry or the possibility of combining multiple images over the same ground area. This will be important for difficult terrain or areas suffering from strong decorrelation effects. An approach for estimating the benefit of multiple acquisitions on a global scale has been presented, based on the interferometric coherence information. The derived relative height error is in good agreement with the estimation approach presented in Section 2 and allows for a further optimization of future acquisitions for completing the final TanDEM-X DEM.

\section{Acknowledgments}

The TanDEM-X project is partly founded by the German Federal Ministry for Economics and Technology (Förderkennzeichen $50 \mathrm{EE}$ 1035). The authors would like to thank the anonymous reviewers for their valuable comments and suggestions to improve the quality of the paper.

\section{References}

Fritz, T., Rossi, C., Yague-Martinez, N., Rodriguez-Gonzalez, F., Lachaise, M., Breit, H. 2011. Interferometric performance of TanDEM-X data. In: Proc. Geoscience and Remote Sensing Symposium (IGARSS), Vancouver, 24-27 July, pp. 2428-2431.

GLOBCOVER, 2009. European Space Agency GLOBCOVER. <http:// ionia1.esrin.esa.int/> (accessed 02.12).

Hueso Gonzalez, J., Bachmann, M., Hofmann, H., 2010. TanDEM-X commissioning phase status. In: Proc. Geoscience and Remote Sensing Symposium (IGARSS), Honolulu, 25-30 July, pp. 2633-2635.

Hueso Gonzalez, J., Bachmann, M., Krieger, G., Fiedler, H., 2010. Development of the TanDEM-X calibration concept: analysis of systematic errors. IEEE Transactions on Geoscience and Remote Sensing 48 (2), 716-726.

Kraus, T., Schrank, D., Rizzoli, P., Bräutigam, B., 2011. In-orbit SAR Performance of TerraSAR-X and TanDEM-X satellites. In: 12th URSI commission - triennial open symposium on wave propagation and remote sensing, GarmischPartenkirchen, 8-11 March.

Krieger, G., Moreira, A., Fiedler, H., Hajnsek, I., Werner, M., Younis, M., Zink, M., 2007. TanDEM-X: a satellite formation for high-resolution SAR interferometry. IEEE Transactions on Geoscience and Remote Sensing 45 (11), 3317-3341.

Lachaise, M., Eineder, M., Fritz, T., 2007. Multi baseline SAR acquisition concepts and phase unwrapping algorithms for the TanDEM-X mission. In: Proc. Geoscience and Remote Sensing Symposium (IGARSS), Barcelona, 23-27 July, pp. 52725276 .

Lee, J.-S., Hoppel, K.W., Mango, S.A., Miller, A.R., 1994. Intensity and phase statistics of multilook polarimetric and interferometric SAR imagery. IEEE Transactions on Geoscience and Remote Sensing 32 (5), 1017-1028.

Martone, M., Rizzoli, P., Bräutigam, B., Krieger, G., 2011. First interferometric performance results of TanDEM-X commissioning phase. In: 12th URSI 
Commission - Triennial Open Symposium on Wave Propagation and Remote Sensing, Garmisch-Partenkirchen, 8-11 March.

Martone, M., Bräutigam, B., Rizzoli, P., Gonzalez, C., Bachmann, M., Krieger, G., this issue. Coherence evaluation of TanDEM-X interferometric data. ISPRS Journal of Photogrammetry and Remote Sensing.

Rodríguez, E., Morris, C.S., Belz, J.E., 2006. A global assessment of the SRTM performance. Photogrammetric Engineering \& Remote Sensing 72 (3), 249-260.

Schwerdt, M., Hueso Gonzalez, H., Bachmann, M., Schrank, D., Döring, B., Tous Ramon, N., Walter Antony, J.M., 2011. In-orbit calibration of the TanDEM-X system. In: Proc. Geoscience and Remote Sensing Symposium (IGARSS), Vancouver, 24-27 July, pp. 2420-2423.
USGS GTOPO, 2001. GTOPO30 Global Digital Elevation Model. <http://eros.usgs.gov/ \#/Find_Data/Products_and_Data_Available/gtopo30_info> (accessed 06.12).

Werner, M., 2001. Shuttle radar topography mission (SRTM): mission overview. Frequenz 55 (3-4), 75-79.

Werninghaus, R., Buckreuss, S., 2010. The TerraSAR-X mission and system design IEEE Transactions on Geoscience and Remote Sensing 48 (2), 606-614.

Wessel, B., 2011. TanDEM-X Ground Segment DEM Products Specification Document. DLR Doc. TD-GS-PS-0021, Issue 1.7, Date 4.11.2011. 